\title{
Why don't patients attend the asthma clinic?
}

\author{
K Gruffydd-Jones, I Nicholson, L Best and E Connell
}

Kevin Gruffydd-Jones General Practitioner

\section{Isla Nicholson}

Practice Nurse

Lynda Best

Practice Nurse

\section{Elizabeth Connell}

Practice Nurse

Box Surgery, Box,

Wiltshire SN13 9NA, UK

Correspondence to:

Dr K Gruffydd-Jones

middlehill@gruffbox.f9.co. uk

Date received: 19/01/99

Date accepted: 04/08/99

\section{ABSTRACT}

Aims: To find out why asthma patients do not attend for structured nurse-led asthma care in a general practice. A secondary aim was to look at some of the morbidity characteristics of these patients.

Method: A telephone questionnaire was administered by practice nurses to asthma clinic nonattenders (to parents of non-attenders age 5-16).

Results: Out of 578 asthmatic patients over the age of 5 (practice list $=6300$ ), 357 were 'non-attenders'. Of these, $225(63 \%)$ perceived that they no longer had asthma or that their asthma was not serious enough to warrant a routine checkup. 106 patients $(30 \%)$ saw their own GP instead.

Logistical reasons for nonattendance such as timing of appointments and difficulty with transport accounted for less than $6 \%$ of patients. The 'low perceivers' had significantly lower morbidity, night-time waking and oral steroid usage than the group as a whole, but the patients only seeing their GP exhibited significantly higher morbidity for the same parameters.

Conclusions: In our asthma-interested practice there are a high number of asthma clinic nonattenders. The main reasons for non-attendance are low perception of asthma severity and patients seeing their own GP. The latter group have a relatively higher level of asthma morbidity. These results suggest that asthma management in primary care needs to involve all doctors in a practice and cannot be left to the asthma clinic alone.

\section{INTRODUCTION}

In recent years, there has been a move in general practice towards structured nurse-run asthma care. Such care can improve quality of asthma management and reduce asthma morbidity. ${ }^{1,2}$ However, many asthma patients do not attend for formalised care, even when this is available. ${ }^{3}$

Box Surgery is a semi-rural practice, with 6300 patients. The practice has been operating structured asthma care since 1986. Initially in the form of a specific clinic, it has now evolved into a system of dedicated asthma appointments with one of two National Asthma and Respiratory Training Centre (NARTC) diploma-level asthma nurses, working to a practice protocol and supported by an asthmainterested general practitioner (GP).
Table 1 Reasons for non-attendance

\begin{tabular}{lcc} 
Reasons for non-attendance & $\begin{array}{c}\text { Number of } \\
\text { Patients }\end{array}$ & $\begin{array}{c}\text { Percentage of } \\
\text { non-attenders }\end{array}$ \\
\hline 'Haven't got asthma' & 119 & $33 \%$ \\
\hline 'Asthma not serious enough' & 106 & $30 \%$ \\
\hline See own GP instead & 106 & $30 \%$ \\
\hline Couldn't see any point in attending & 49 & $14 \%$ \\
\hline Don't use prescribed medicines/inhalers & 45 & $13 \%$ \\
\hline Timing of appointments inconvenient & 17 & $5 \%$ \\
\hline Forgot & 12 & $3 \%$ \\
\hline Attend hospital only & 3 & $1 \%$ \\
\hline Other reasons e.g. no car/housebound & 2 & $61 \%$ \\
\hline Not stated & 23 & $6 \%$ \\
\hline
\end{tabular}

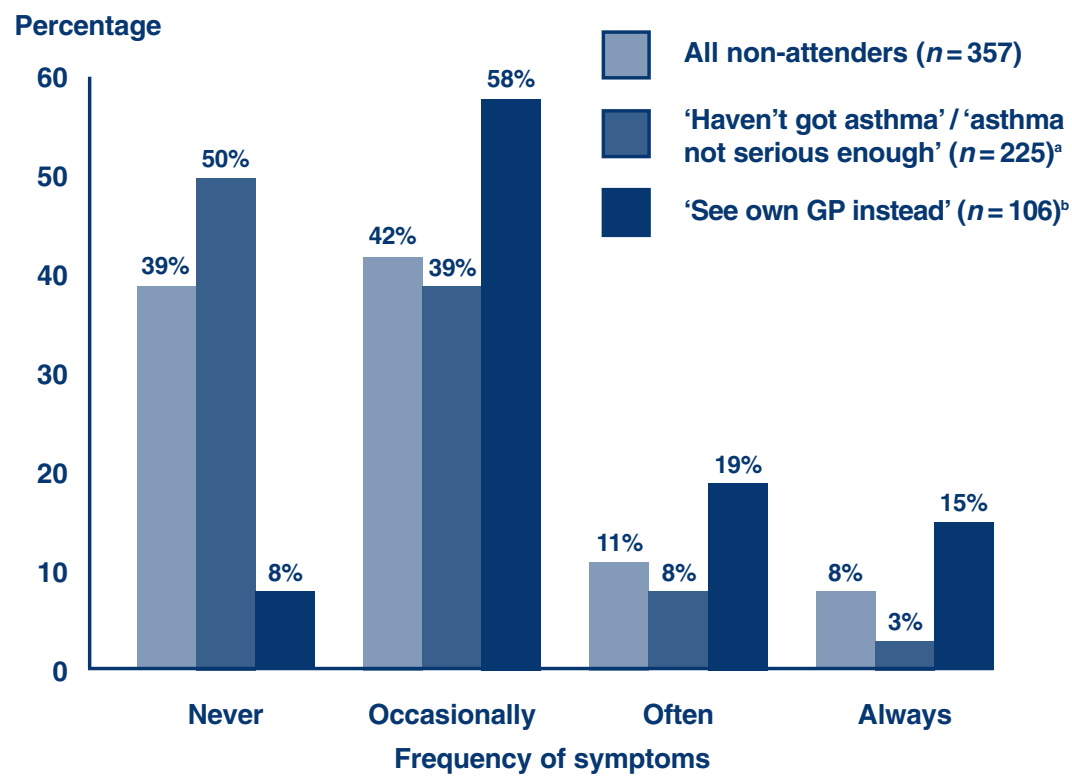

Figure 1 How often patients experience cough, wheeze or shortness of breath

a. $\chi^{2}$ trend compared to rest of non-attenders $=28.6(p<0.001)$

b. $\chi^{2}$ trend compared to rest of non-attenders $=5.45(p<0.025)$ 
A study was performed to find out why asthma patients do not attend for structured asthma care in our practice (the term 'clinic' will be used to indicate nurse-led structured asthma care). A secondary aim was to look at some of the morbidity characteristics of these patients.

\section{METHOD}

Non-attenders were identified from the practice asthma register. The study was carried out by telephone questionnaire, by one of two practice nurses, between $1 / 2 / 97$ and $1 / 8 / 97$. Telephone calls were made opportunistically to implement a structured questionnaire to patients over the age of 16 (or to parents of children age 5-16) who had not attended the asthma clinic in the previous 12 months.

Patients were asked about the reasons for nonattendance at the asthma clinic and about some indicators of asthma morbidity including:

1. Frequency of symptoms such as cough, tight chest and shortness of breath

2. Frequency of night-time waking due to asthma in the previous month

3. Courses of oral steroids in the previous year

4. Acute hospital attendances/admissions in the previous year

5. Degree of restriction of daily activities.

Differences between the two main sub-groups of nonattenders, the 'low perceivers' and patients only seeing their GP, were analysed using the chi square trend test. $^{4}$

\section{RESULTS}

578 patients over the age of five were identified on the asthma register. Of these, 221 (38\%) had attended the practice asthma clinic in the last 12 months and 357 $(62 \%)$ were non-attenders.

The reasons for non-attendance are outlined in Table 1 (patients were allowed to answer more than once hence the totals exceed 100\%). $119(33 \%)$ patients stated that they didn't have asthma and $106(30 \%)$ that their asthma wasn't serious enough to attend. A further $106(30 \%)$ patients said that they saw their own GP instead of attending the clinic. Logistical reasons such as timing of appointments or difficulty with transport accounted for less than $6 \%$ of patients.

Morbidity characteristics of the non-attenders are shown in Figures 1-5. The 357 non-attenders exhibited low levels of asthma morbidity, indicated by the low usage of oral steroids (90\% had no courses in the last 12 months) and acute hospital attendances (96\% had none in the last 12 months), but higher symptom levels (19\% experienced symptoms often or always). However, two major sub-groups emerged (see Discussion): a low morbidity 'low perceiver' group and a high morbidity group who saw their own GP only for asthma care ('GP only attenders').

\section{DISCUSSION}

The main reasons for non-attendance at our asthma clinic were the patient's (or parent's) low perceptions of their asthma severity, rather than logistical reasons such as timing of appointments and difficulties with transport. A further $30 \%$ of patients visited their

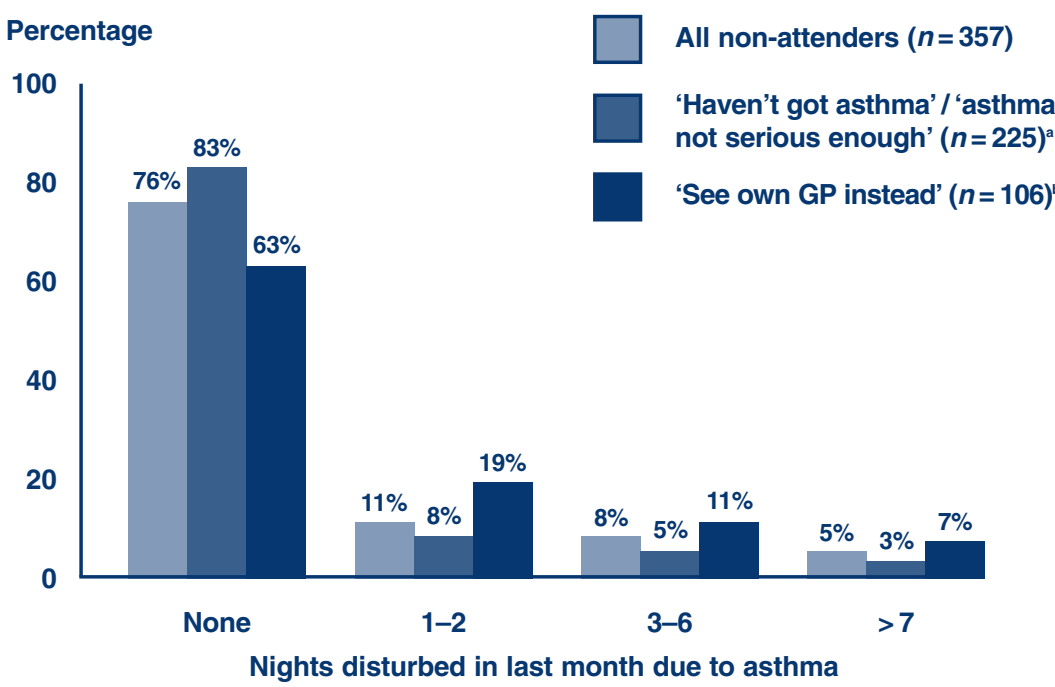

Figure 2 Night disturbance due to asthma in last month

a. $\chi^{2}$ trend compared to rest of non-attenders $=21.9(p<0.001)$

b. $\chi^{2}$ trend compared to rest of non-attenders $=4.85(p<0.05)$

Percentage

All non-attenders $(n=357)$

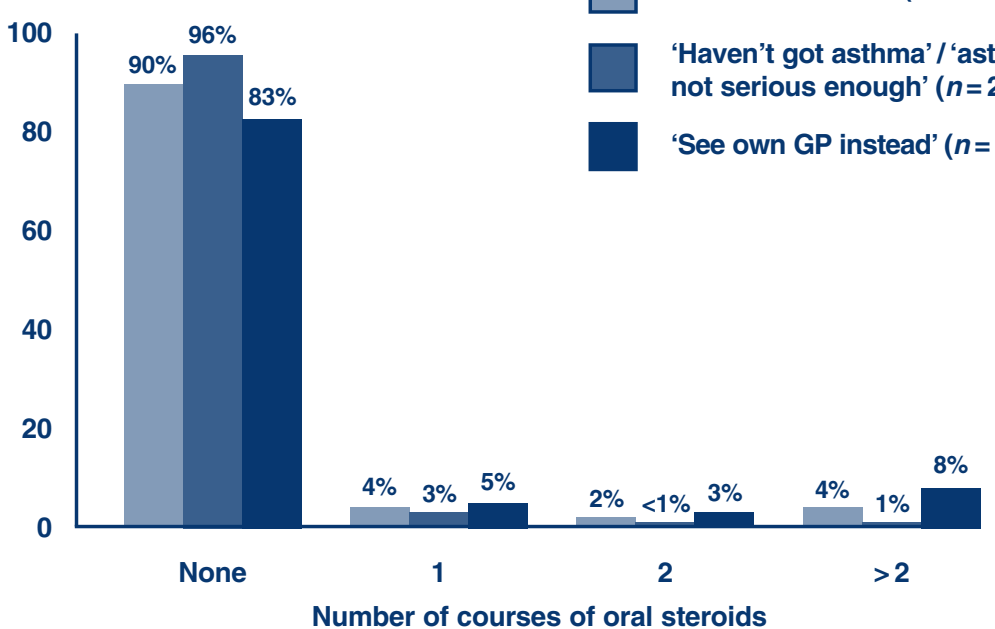

Figure 3 Number of courses oral steroids in last 12 months

a. $\chi^{2}$ trend compared to rest of non-attenders $=25.7(p<0.001)$

b. $\chi^{2}$ trend compared to rest of non-attenders $=23.3(p<0.001)$

own GP instead. The responses to our questionnaire may have been biased by the fact that the practice nurse administered it; patients were probably less likely to criticise the overall running of the clinic. It may have been better to conduct the questionnaire using an independent interviewer.

Overall the impact of asthma amongst non-attenders on everyday life appears to be relatively small, $89 \%$ of patients had no restriction in daily activities, $96 \%$ had not attended hospital and $90 \%$ had not received oral steroids. However, according to symptomatology, there was a higher level of morbidity; $19 \%$ of patients exhibiting symptoms 'often or always' and $23 \%$ waking at least one night in the previous month because of asthma. 


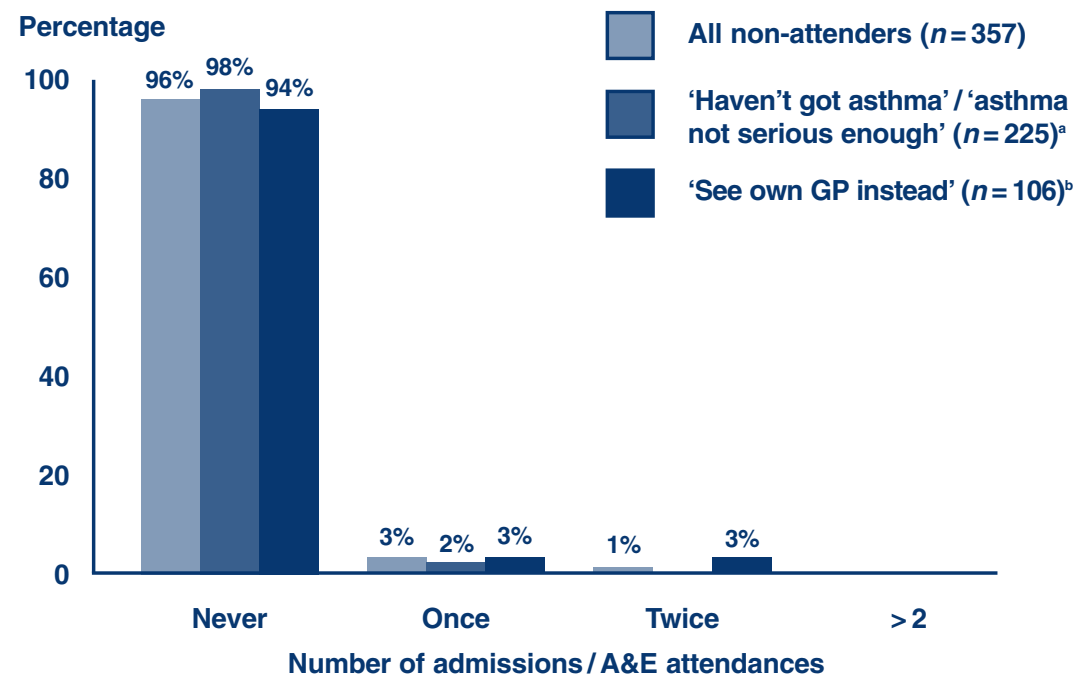

Figure 4 Number admissions / A\&E attendances in last 12 months

a. $\chi^{2}$ trend compared to rest of non-attenders $=0.74$ (NS)

b. $\chi^{2}$ trend compared to rest of non-attenders $=1.33$ (NS)

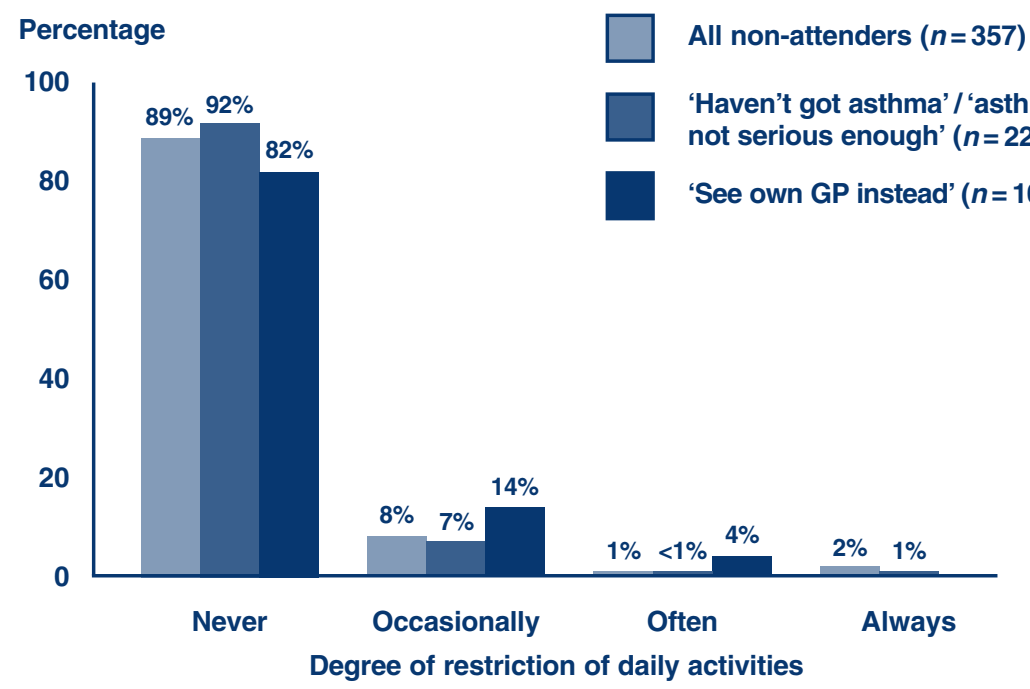

Figure 5 Degree of restriction of daily activities

a. $\chi^{2}$ trend compared to rest of non-attenders $=9.87(p<0.005)$

b. $\chi^{2}$ trend compared to rest of non-attenders $=1.82$ (NS)

This study does not compare the morbidity data of non-attenders and clinic attenders. However, data collected from clinic record cards suggest that morbidity is lower in clinic attenders.

It appeared that the morbidity characteristics of the main sub-groups of low perceivers and GP only attenders might differ from the group of non-attenders as a whole. The morbidity trends in the two subgroups were compared with the rest of the main group, using chi-square trend analysis. Any conclusions drawn from this analysis must be tempered by the fact that the study was not designed to test whether there might be a difference between the two sub-groups.

The low perceivers appear to have lower morbidity for all parameters (except hospital visits). This appears to vindicate patients' perceptions about their asthma severity, however, $50 \%$ of patients in this group had experienced asthma symptoms in the last month. Similarly, Waring ${ }^{5}$ found a group of asthmatics on his practice asthma list who had not received any asthma medication over a two-year period. However, over the subsequent year $17.5 \%$ needed asthma treatment. In other words, even low perceivers will need some kind of formalised follow-up.

The patients who only saw their own GP for asthma care appeared to exhibit a high level of asthma morbidity, $28 \%$ patients had experienced more than two nights disturbance because of asthma in the last month and $16 \%$ had at least one course of oral steroids in the last year. It is uncertain whether these patients had a high morbidity because of sub-optimal care or because patients with more severe symptoms perceived that they needed to see a doctor rather than a nurse. The former hypothesis is supported by analysis of patient records; of those attending the GP alone, there was little evidence of structured asthma care such as routine lung function testing or use of self-management plans.

\section{CONCLUSIONS}

This study revealed that, in our asthma-interested practice, a large number of patients don't attend the asthma clinic. The main reasons for non-attendance were patients' perceptions about their asthma severity rather than logistical reasons. This group appears to exhibit a relatively low level of asthma morbidity whilst there is a significant group of patients, with relatively high morbidity, who only see their GP for asthma care. If these findings are common to other practices, all GPs need to be kept updated about asthma management. It is not sufficient to say 'I leave asthma management to the asthma nurse!'

\section{Acknowledgements}

Thanks to Allen \& Hanburys for kindly providing a research grant.

\section{References}

1. Dickinson J, Hutton S, Atkin A. Implementing the British Thoracic Society guidelines: The effect of a nurse-run asthma clinic on prescribed treatment in an English general practice. Resp Med 1998;92(2):264-7

2. Dickinson J, Hutton S, Atkin A, Jones K. Reducing asthma morbidity in the community: The effect of a targeted nurserun asthma clinic in an English general practice. Resp Med 1997;91:634-40

3. Levy ML, Barnes G, Howe M, Neville RG. Why don't patients attend for asthma follow-up? Asthma Gen Pract 1998;6(Suppl 1):S4

4. Kirkwood BR. Essentials of medical statistics. Oxford, Blackwell Scientific Publications, 1988:102-4

5. Waring N. Use of new asthma BTS steps in one general practice - should asthmatics no longer on treatment be followed up? Asthma Gen Pract 1997;5(2):28 